\title{
NuMERICAL STUdies OF TrapezoIdAL Prototype Auditory Membrane (PAM)
}

\author{
Harto Tanujaya ${ }^{1}$, Satoyuki Kawano ${ }^{2}$ \\ ${ }^{1}$ Department of Mechanical Engineering, Tarumanagara University, Jakarta, Indonesia \\ hart_tan18@yahoo.com \\ ${ }^{2}$ Mechanical Science and Bioengineering Department, Osaka University, Japan
}

\begin{abstract}
In this research, we developed numerically a Prototype Auditory Membrane (PAM) for a fully implantable and self contained artificial cochlea. Cochleae are one of the important organs for hearing in the human and animals. Material of the prototype and implant of PAM are made of Polyvinylidene fluoride (PVDF)Kureha, Japan which is fabricated using MEMS and thin film technologies. Another important thing in the characteristic of the PAM is not only convert the acoustic wave into electric signal but also the frequency selectivity. The thickness, Young's modulus and density of the PAM are $40 \mathrm{~m}, 4 \mathrm{GPa}$, and $1.79 \times 10^{3}$ $\mathrm{kg} / \mathrm{m}^{3}$, respectively. The shape and dimension of the PAM is trapezoidal with the width is linearly changed from 2.0 to $4.0 \mathrm{~mm}$ with the length are $30 \mathrm{~mm}$. Numerically, we develop the model of PAM is based on commercial CFD software, Fluent 6.3.26 and Gambit 2.4.6. The geometry model of the PAM consists of one-sided blocks of quadrilateral elements for 2D model and tetrahedral elements for 3 D model respectively. In this study we set the flow as laminar and carried out using unsteady time dependent calculation. The results show that the frequency selectivity of the membrane is detected on the membrane surface.
\end{abstract}

\section{KEYWORDS}

Cochlea, PVDF, PAM, Frequency Selectivity

\section{INTRODUCTION}

As a medical treatment for sensorineural hearing loss in children and adults, cochlear implant is recently used [1][2]. The current cochlear implant consist of an implant stimulating electrodes and an extracorporeal device, which bypass the damaged hair cells by generating electric current in response to the acoustical sound [3]. There is a battery inside the extracorporeal device, which is one of the critical disadvantages in the current cochlear implant. This status motivates us to develop a fully self-contained artificial cochlea.

The function and geometrical of the cochlea has been being studied by many researchers in the world. Measuring and modelling the geometric of the cochlea and biological basilar membrane technically are very complicated, these become a challenge to be researched. In this research we use the Prototype Auditory Membrane (PAM) as the simplifying model of the biological basilar membrane. The important role of the cochlea is not only conversion of acoustical sound to

DOI : $10.5121 /$ ijcsea.2012.2404 
electrical signals but also frequency selectivity [4]. Numerical simulation is one of tools to predict the frequency selectivity on it. The simulation of fluid flow is an application of numerical method for estimating the pattern of fluid flow distribution as well as on cochlea. These methods describe the prediction of fluid flow process and its distribution, before and after a real fluid flow process happened [5]. The information about fluid flow distribution with certain frequency are utilized to create a pressure distribution area map and fluid flow activity. Generally in this research, we have studied in depth the characteristic of the artificial cochlea. We will report a novel piezoelectric artificial cochlea which works on acoustic wave with frequency selectivity. This system is related with the oscillation and piezoelectric effect. Experimentally, the frequency of the acoustic wave is controlled from $1 \mathrm{kHz}$ to $20 \mathrm{kHz}$, which covers the human audible frequency. The research paper consists of an introduction as well as a brief description of the research objective, mechanical model and design including results of the numerical simulation.

\section{NUMERICAL SimULATION}

In this paper, we develop and simulate the model is based on the numerical method. The model is developed and simulated based on the commercial CFD software, Fluent 6.3.26, and Gambit 2.4.6. The Fluent 6.3.26 use finite-volume code, generally this commercial software is used in hydrodynamics and mass transfer computations [6]. The Gambit 2.4.6 is used to provide the complete mesh flexibility, and solve the flow problems with both structured and unstructured meshes. All functions of the Fluent and Gambit software's are required to compute a solution and display the results. These software are also accessible either through an interactive interface or constructed using user-defined-functions (UDS).

The main challenge of the numerically predicting sound waves stems from the well-recognized fact that sounds have much lower energy than fluid flows, typically by several orders of magnitude. This poses a great challenge to the computation of sounds in terms of difficulty of numerically resolving sound waves, especially when one is interested in predicting sound propagation to the far field.

\subsection{CFD}

At present, Computational Fluid Dynamic (CFD) is a relatively new engineering tool that has grown side by side with the development of the computer. CFD has been limited only by computer RAM or lack of there, due to the use on billions upon billions of calculations and iterations. Fluent was used in this project, this software was used to simulate flow in very small duct (MEMS) and determine the pressure that occur in the duct with reasonable accuracy. This is key to many fields of engineering because duct flows similar to this are used by the medical, automotive, horticultural, and construction industries. As a result, equations and plots can be created to assist industry in the production of better products.

It is the capability to develop larger and more complex simulations of real world fluid experiments on computers. Generally, computers can be used to solve FEA stress simulations, simulate fluid flow and heat transfer. Along with computers, the Navier-Stokes equation allowed large, complex simulations of fluid flows to be solved. Equations below are the Navier-Stokes equation [7]. 
Surprisingly, this equation was developed in the early nineteenth century by Claude Louis Marie Navier and Sir George Gabriel Stokes. In CFD, the differential equations are used as algebraic equations that describe small finite fluid elements that can then be estimated.

CFD simulation usually includes three steps: preprocessing, processing, and post processing. For the preprocessing step, a specific system is identified. The geometry and material properties should be clearly defined, and meshing usually follows after geometry is determined. This is accomplished by dividing geometry into many small elements or volumes. Meshing in Gambit is a complicated work, as it is critical for both the accuracy of final result and the cost of numerical calculation. Generally, the finer the divided elements are the closer will be the final solution to the true value at the expense of more calculation time. The setting of boundary conditions, initial conditions and convergence criteria are also completed in the pre-processing stage. In the processing stage, there are iterative calculations in each cell, which are carried out until convergence criteria are met. This calculation-intensive process constitutes is the core content of CFD application. It is not uncommon to have tens of thousands of calculation for even a relatively simple problem. After the completion of processing, the results can be evaluated either numerically or graphically. The graphical methods provide a more convenient way to evaluate the overall effect. This includes signal plot, frequency, vector plot, plot of scalar variables, etc. These visualization tools in post-processing stage allow quick assessment and comparison of calculation results.

This research utilized a CFD program called Fluent. Fluent and CFD programs make guesses at the solution and check how accurate the guess until it reaches a user defined "convergence." The convergence term is similar to an error value that results from the computers guess at the NavierStokes equation and represents the precision of the simulation. Many of the Navier-Stokes variables are input by the user such as the inlet pressures, boundaries, outlet types, and fluid properties. Next, the computer makes a guess at the velocity field for the system and checks the accuracy of its guess [8].

This cycle is referred to as a iteration. Iterations occur until the computers guesses reach the user defined convergence point. Once that is accomplished, the user can examine the velocity field for flow rates, pressures, energy transfers, and many other important characteristics. For this project, the pressure into and out of the system was examined. However to use Fluent, a finite element grid or mesh of the geometry was needed.

Packaged with Fluent is a program called Gambit. Gambit is a CAD (computer aided design) program that allows a user to generate a model and then section it into small control volumes for use in Fluent. The accuracy of the Fluent simulations are critically tied to this program because Gambit is responsible for how many analysis points are placed in the model. Simply, put the higher number of volumes, because the higher volumes can make more the accurate. This also has a down side because with more volumes, comes longer iteration time. Moreover, this can sometimes exceed the computers available memory and computational abilities.

This research explores the behavior of a fluid that is subjected to a force and the flow pattern that is resulted. Basic mechanisms need to be understood before Fluent can be used because Fluent requires the user to input the external constraints and set up the simulation environment. To produce a fluid flow, a difference in pressure between the inlet and each outlet is required. 
The governing equations of the sound waves through the duct are the mass continuity equation, equilibrium equation and energy equation. Generally, the equation of motion governs fluid flow is well known as the Navier-Stokes equation. Most researches on fluid dynamics are mostly dedicated to get the solutions of this equation with particular boundary conditions, because of difficulties in obtaining exact solutions for this kind of nonlinear equation [9].

The fluid characteristic by two parameters, fluid velocity and fluid density and the behavior of fluid flow obeys two laws, for instance the conservation of mass and the conservation of momentum. The conservation of mass means the initial and the final masses should remain the same. Let consider a finite volume $(\forall)$ of fluid with A is a closed surface of the finite volume. The mass of fluid using a finite volume is $\int_{c v} \rho d \forall$, the mass of fluid through a closed surface is $\int_{c S} \rho d \forall$. The conservation of mass means that the incoming and outgoing flux of fluid are conserved per unit time in a finite volume.

\subsection{Two and Three Dimensional Model of PAM}

The grid model of PAM with a basiliar membrane is shown in Figure 1. The PAM is one of Microelectomechanical System (MEMS) device. In this study, the frequency selectivity of the PAM can be investigated using computational fluid dynamics (CFD) and structural analysis. Simulation model of the PAM consists of two cochlear ducts both filled with liquid. In the middle of the ducts there is a basilar membrane. The membrane separates the ducts as shown in Figure 1. The shape of basilar membrane is trapezoidal and the mechanical impedance changes along the longitudinal direction. The acoustic pressure is applied to the liquid from the inlet region, the membrane will be oscillated due to the fluid-structure interaction and it works as a so-called variable wave guide. In order to clarify the characteristics of frequency selectivity, the fluid flow and the vibration of the membrane are studied based on the numerical simulation.

We use the 2D and 3D models of the artificial cochlear in this calculation. The inlet position of 2D and 3D model are located at side of the membrane, respectively. The flow in the cochlear ducts modeled as Newtonian, compressible, laminar and unsteady liquid flow based on the Navier-Stokes equations. Surface of the membranes are treated as non-slip and rigid wall. Equation of state relate the other variables to the two state variables, if we use $\rho$ and $T$ as state variables we have state equations for pressure $p$ and specific internal energy $i, p=p(\rho, T)$ and $i$ $=\mathrm{i}(\rho, \mathrm{T})$, for perfect gas the following, equation of state are useful, $p=\rho \mathrm{RT}$ and $\mathrm{i}=\mathrm{c}_{\mathrm{v}} \mathrm{T}[6]$.

$$
p_{\text {abs }}=p_{o p}+p_{\text {gauge }} \quad \rho=\frac{p_{o p}+p_{\text {gauge }}}{R_{o} . T}
$$

The compressible fluid flows, the equations of state provide the linkage between the energy equation on the one hand, and mass conservation and momentum equations on the other. Without density variation there is no linkage between the energy equation and the mass conservation and momentum equations. The basic equations are numerically solved using Fluent 6.3.26.

Acoustic pressure pulse applied from the inlet to effectively analyze the response at various frequencies. The width and the magnitude of the pulse define are $20 \mathrm{~s}$ and $0.002 \mathrm{~Pa}$, respectively, where the pulse includes waves at all of the audible frequencies from $20 \mathrm{~Hz}$ to 20 $\mathrm{kHz}$. The spatial distribution of acoustic pressure at a certain frequency obtained from the frequency spectrum of the acoustic pressure. 
The meshing work for the geometry of the duct was done with Gambit 2.4.6 in 3-D analyses. Grid refinement was performed according to the concentration gradient within the module geometry. The final result represented the result after completing grid refinement and grid independence. The pressure and momentum discretization schemes are second-order accurate, for time integration process, first order implicit method used [14].

In this paper, we develop the model of artificial cochlea using plat bending theory and simulation were based on the commercial CFD software, Fluent 6.3.26 and Gambit 2.4.6. The geometry of the duct consist of 1 one-sided blocks of quadrilateral elements for 2D model and tetrahedral elements for 3D model respectively, using map-type mesh and was exported to Fluent ${ }^{\circledR}$. In this block, there are 31,903 nodes, 63,222 faces for 2D model and 188,796 nodes, 2,015,880 faces for 3D model. In this study we set the flow as compressible flow and we do not use the turbulence flow because the exploration times are relatively short. Thus we consider the following assumptions related to the fluid flow domain, the fluid is a continuous medium, Newtonian, gravity is neglected and the flow is laminar. There are three positions of measurement, inlet, middle and outlet regions as shown in Figure 1 (a) (c).

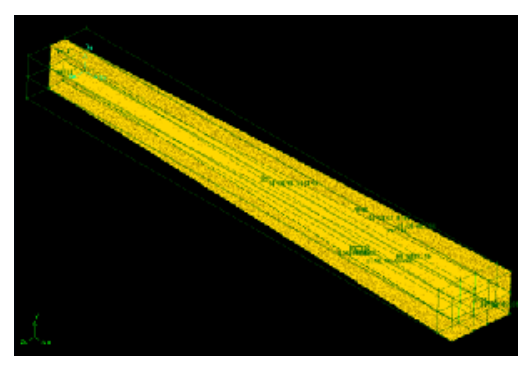

(a) Trapezoidal Grid Design

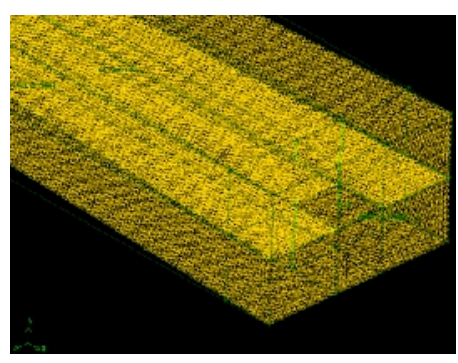

(b) Basal Grid

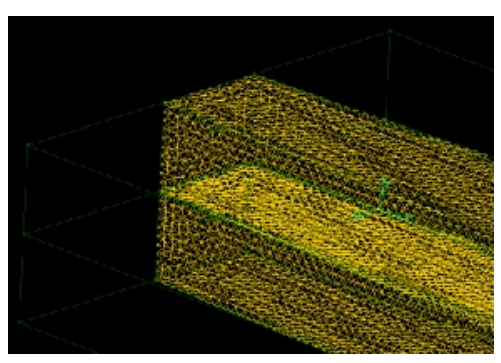

(c) Apex Grid

Figure 1 Grid structure (a) Trapezoidal grid design (b) basal grid (c) apex grid

Figure 1 and 2 show the mesh and the grid of the duct model with the basilar membrane in the middle and center of the cochlear duct.

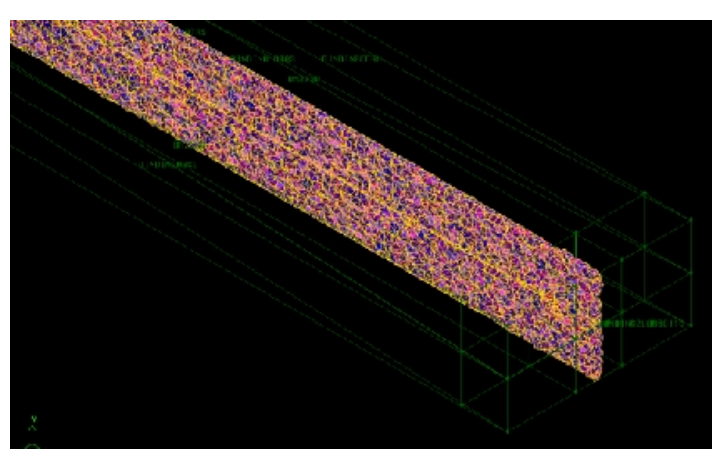

(a) Part

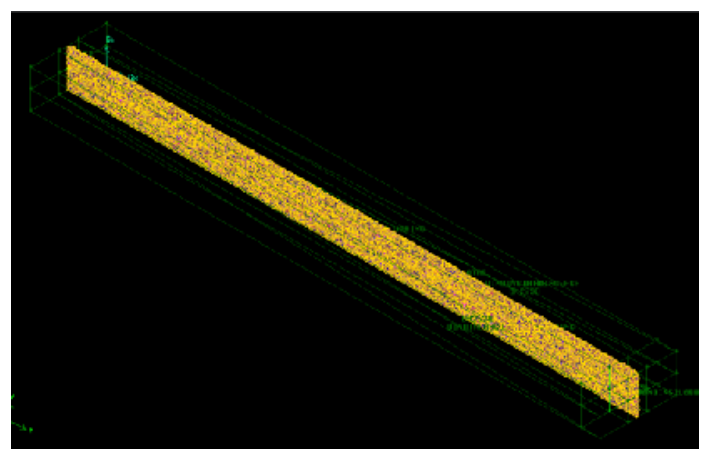

(b) Full

Figure 2. Mesh at the center of the duct model (a) part (b) full

The volume statistic for the modeling and simulations are shown, 
Table 1. Volume statistic for modeling and simulations

\begin{tabular}{|c|c|c|}
\hline Type & Grid check 2D Model & Grid check 3D Model \\
\hline $\begin{array}{l}\text { Domain Extents: } \\
\text { - x-coordinate } \\
\text { - y-coordinate } \\
\text { - z-coordinate }\end{array}$ & $\begin{array}{c}\min (\mathrm{mm})=0.000 \mathrm{e}+000 \\
\max (\mathrm{mm})=30 \\
\min (\mathrm{mm})=0.000 \mathrm{e}+000, \\
\max (\mathrm{mm})=20 \\
-\end{array}$ & $\begin{array}{l}\min (\mathrm{mm})=0.000 \mathrm{e}+000 \\
\max (\mathrm{mm})=30.000 \mathrm{e}+000 \\
\min (\mathrm{mm})=0.000 \mathrm{e}+000 \\
\max (\mathrm{mm})=20.000 \mathrm{e}+000 \\
\min (\mathrm{mm})=0.000 \mathrm{e}+000 \\
\max (\mathrm{mm})=40.000 \mathrm{e}+000\end{array}$ \\
\hline $\begin{array}{l}\text { Volume Statistic: } \\
\text { • min. volume } \\
\text { • max volume }\end{array}$ & $\begin{array}{l}2.448890 \mathrm{e}-007(\mathrm{~mm} 2) \\
7.943498 \mathrm{e}-003(\mathrm{~mm} 2)\end{array}$ & $\begin{array}{l}1.571 \mathrm{e}-006(\mathrm{~mm} 3) \\
4.560 \mathrm{e}-004(\mathrm{~mm} 3)\end{array}$ \\
\hline $\begin{array}{l}\text { Face area statistics : } \\
\text { - min face area } \\
\text { - max face area }\end{array}$ & $\begin{array}{l}2.000000 \mathrm{e}-005(\mathrm{~mm}) \\
4.766194 \mathrm{e}-001(\mathrm{~mm})\end{array}$ & $\begin{array}{l}8.319 \mathrm{e}-005(\mathrm{~mm} 2) \\
1.339 \mathrm{e}-002(\mathrm{~mm} 2)\end{array}$ \\
\hline Residual Criteria & $\begin{array}{l}\text { 1e-005 (continuity, velocity) and } \\
\text { 1e-006 (Energy) }\end{array}$ & $\begin{array}{l}\text { 1e-005 (continuity, velocity) and } \\
\text { 1e-006 (Energy) }\end{array}$ \\
\hline
\end{tabular}

Water-liquid is used in this simulation, this assumption based on the liquid fluid inside the cochlea is endolymph that almost same with water characteristic. The detail information about this fluid as shown in table below,

Material Properties,

Table 2. Characteristic of fluid : water-liquid (fluid)

\begin{tabular}{|l|c|c|c|}
\hline \multicolumn{1}{|c|}{ Property } & Units & Method & Value(s) \\
\hline Density & $\mathrm{kg} / \mathrm{m} 3$ & user-defined & (superfluid_density) \\
\hline Cp (Specific Heat) & $\mathrm{j} / \mathrm{kg}-\mathrm{k}$ & constant & 4182 \\
\hline Thermal Conductivity & $\mathrm{w} / \mathrm{m}-\mathrm{k}$ & constant & 0.60000002 \\
\hline Viscosity & $\mathrm{kg} / \mathrm{m}-\mathrm{s}$ & constant & 0.001003 \\
\hline Molecular Weight & $\mathrm{kg} / \mathrm{kgmol}$ & constant & 18.0152 \\
\hline Speed of Sound & $\mathrm{m} / \mathrm{s}$ & user-defined & (sound_speed) \\
\hline
\end{tabular}

Assumption in this simulation are,

- Fluid : Water - H20 (liquid)

- Laminar Flow

- Density : UDF (Tait Equation)

- Pressure Inlet : $0.002 \mathrm{~Pa}$ - for 20e-006 s

- Number of time step : 400

- BM model : Non-slip and Rigid wall 
- Bulk Modulus (water) : 2.2E+09 Pa

- Density water (at $1 \mathrm{~atm}): 1000 \mathrm{~kg} / \mathrm{m} 3$

- Pressure Operating : $101325 \mathrm{~Pa}$

- Temperature Operating : $300 \mathrm{~K}$

The criteria for convergence were $1.0 \times 10^{-5}$ for continuity, velocity and energy. The time step size is determined to be $2.0 \times 10^{-6} \mathrm{~s}$ and the total calculation time is $2.0 \times 10^{-3} \mathrm{~s}$.

- Input data :

- 10 time step with 0 Pa for $2.0 \times 10^{-5}$ seconds

- 10 following time step using $0.002 \mathrm{~Pa}$ for $2.0 \times 10^{-5}$ seconds

- 980 following time step using 0 Pa until $2.0 \times 10^{-3}$ seconds

\section{RESULTS AND DISCUSSION}

In this paper, the liquid in the duct model is modeled as non-viscous fluid. The flow is a laminar flow model which was controlled by pressure on the pressure-inlet option. The simulation models are 2 and 3 dimensional square mesh with cartesian coordinate. The density of the cochlear fluids that was filled into the duct is estimated using UDF. The simulation process is carried out using unsteady time dependent calculation.

Figure 3 show the pressure distribution at inlet region of $2 \mathrm{D}$ model. In this figure the pressure was given of 2.00E-05 seconds, and the simulation running for 8.00E-04 seconds.

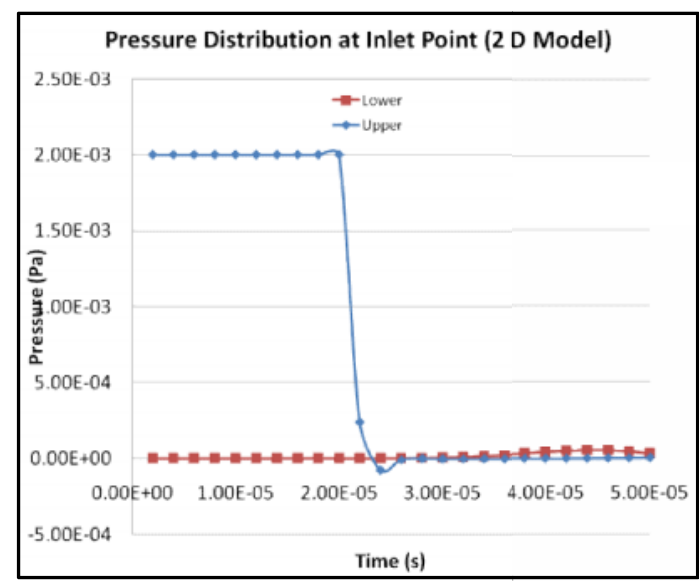

(a) Upper

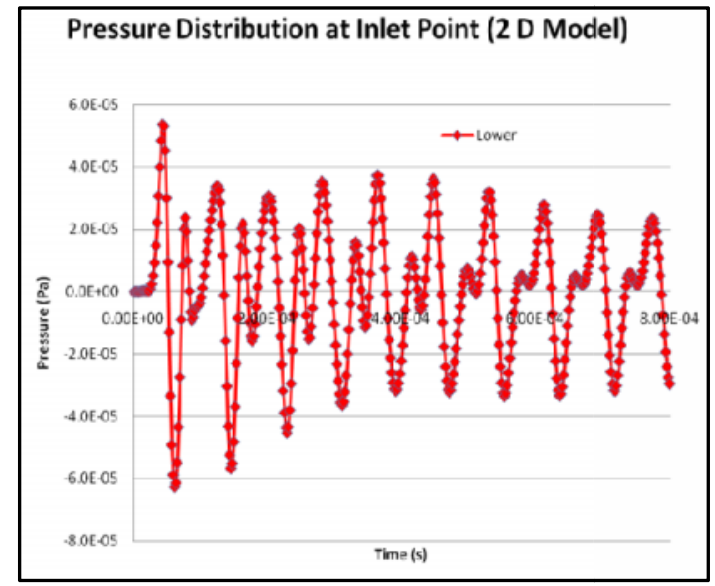

(b) Lower

Figure 3. Pressure distribution at inlet point 2D model (a) upper (b) lower

Figure 4 show the pressure distribution at inlet region of 3D model. The pressure is gave with same with 2Dmodel of 2.00E-05 seconds. Based on the Figure 3 and 2, the magnitude of the pressure are explained that $2 \mathrm{D}$ model has bigger magnitude than $3 \mathrm{D}$ model, but generally the both pressure distribution of 2D and 3D model has almost the similar forms. 


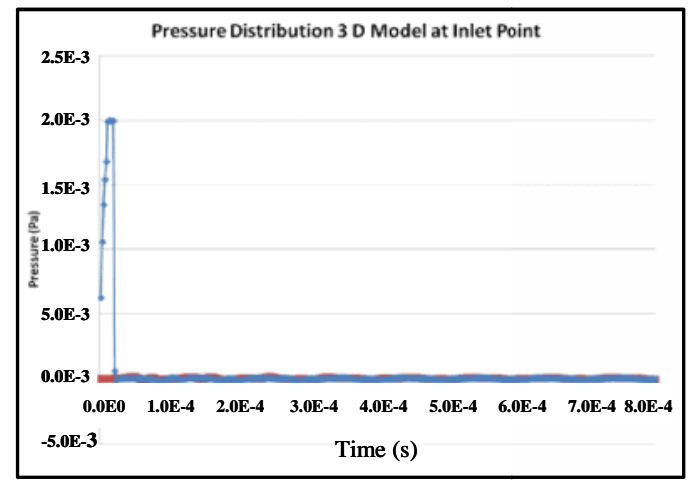

(a) Upper

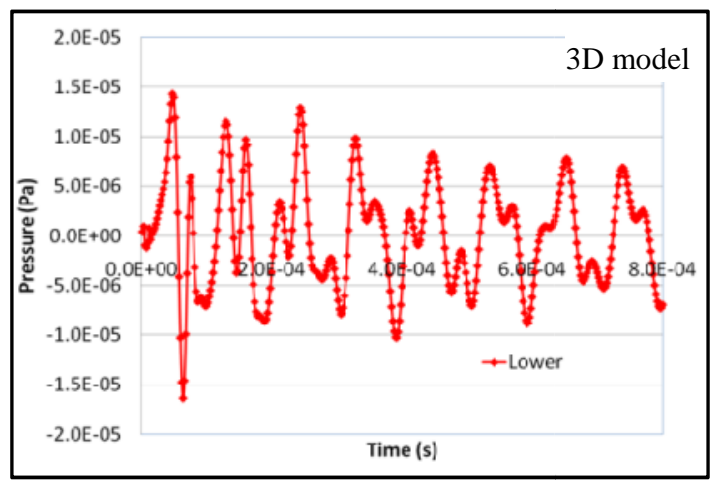

(b) Lower

Figure 4. Pressure distribution at inlet point 3D model (a) upper (b) lower

Figure 5 show the pressure distribution of 2D and 3D model at the center point of the membrane. In the figure, magnitude of the pressure distribution using 3D model has lower than 2D model. The wavelength of the 2D model is shorter than 3D model, so the frequency at the center point of $2 \mathrm{D}$ model is higher than $3 \mathrm{D}$ model at the same conditions.

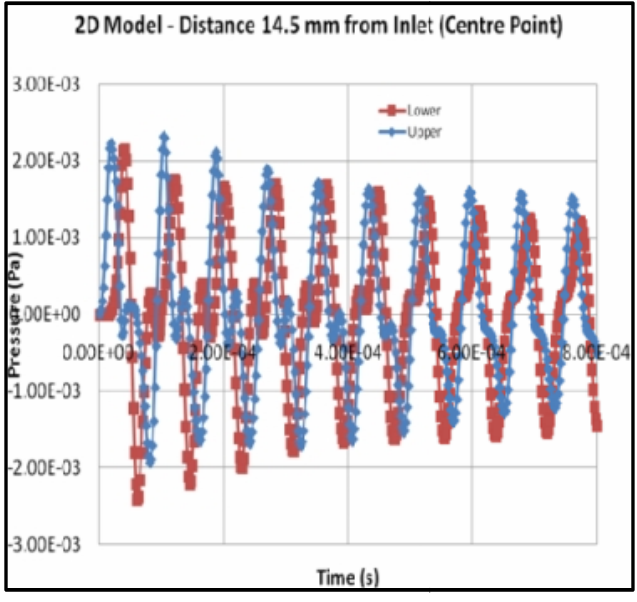

(a) 2D Model

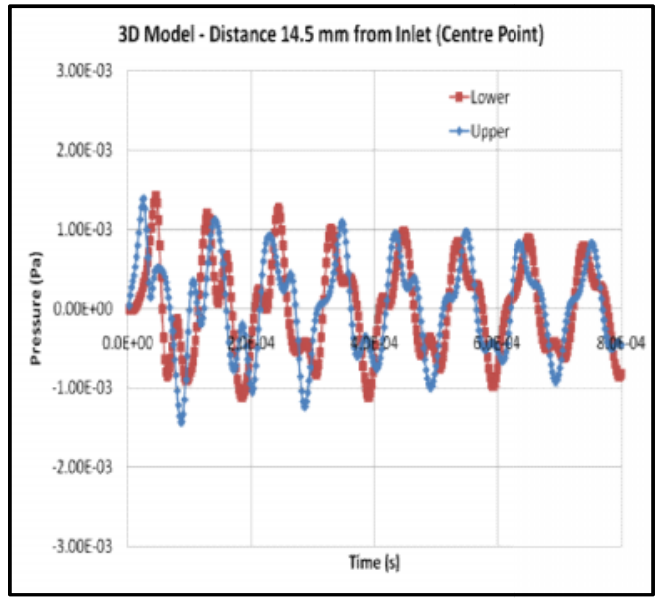

(b) 3D Model

Figure 5. Pressure distribution at distance $14.5 \mathrm{~mm}$ (a) 2D and (b) 3D model

Figure 6 show the pressure distribution of 2D and 3D model at the end point of $29 \mathrm{~mm}$ from base point. 2D model has more period than 3D, this indicate that analysis using 2D model has higher frequency than 3D. It is caused by design of the model as dimension and amount of the grid different each other. The both graph of 2D and 3D showed to the periodical in oscillation with the value of the fluctuation that was not too big and the amplitude was tended to decrease exponentially. 
International Journal of Computer Science, Engineering and Applications (IJCSEA) Vol.2, No.4, August 2012

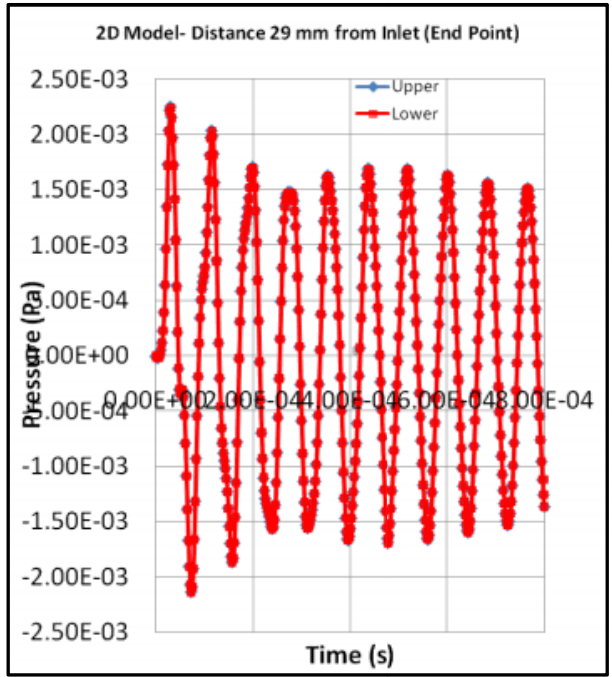

(a) 2D Model

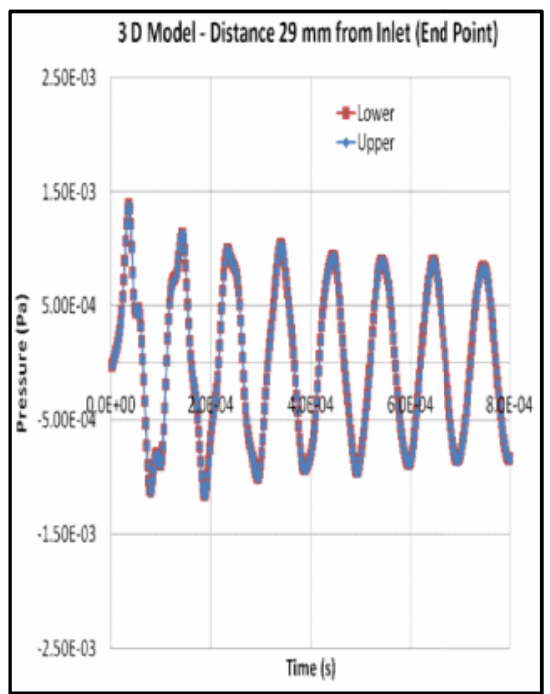

(b) 3D Model

Figure 6. Pressure distribution at distance $29 \mathrm{~mm}$ of (a) 2D and (b) 3D model

Figure 7 show spectral density of 2D model at (a) inlet - upper (b) inlet - lower (c) center point (d) end point. Frequency at the inlet-upper, inlet-lower, center point, and end point of the membrane are occurred at the same frequency of $12.6 \mathrm{kHz}$. This indicates that the analysis numerical of the membrane using 2D model has resonance frequency around $12.6 \mathrm{kHz}$.

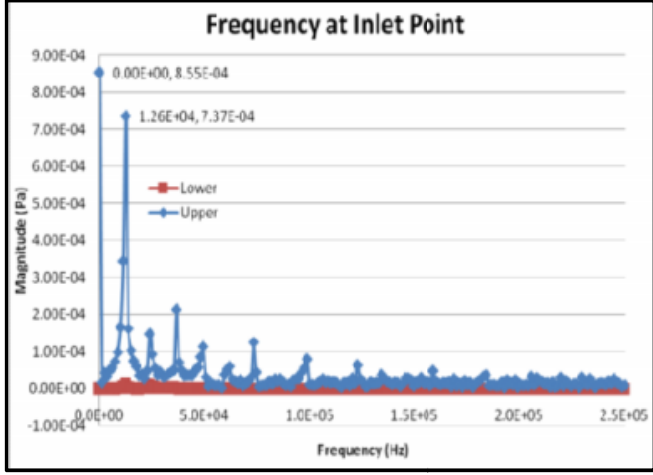

(a) Inlet - Upper

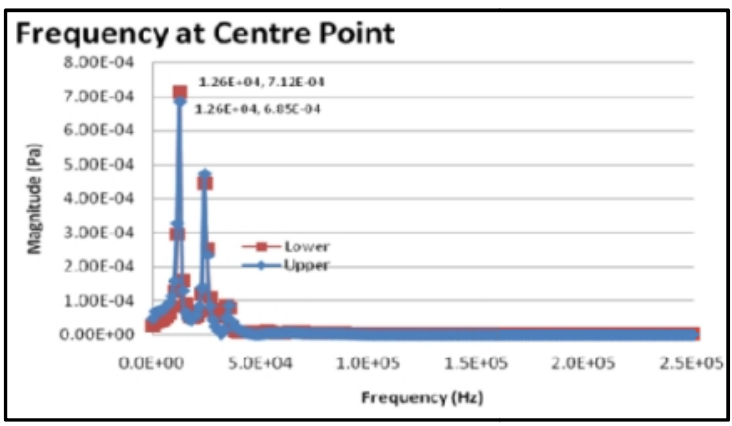

(c) Center Point

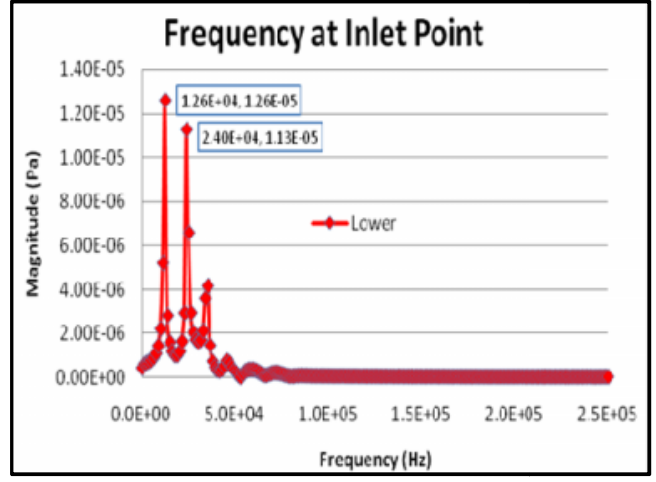

(b) Inlet - Lower

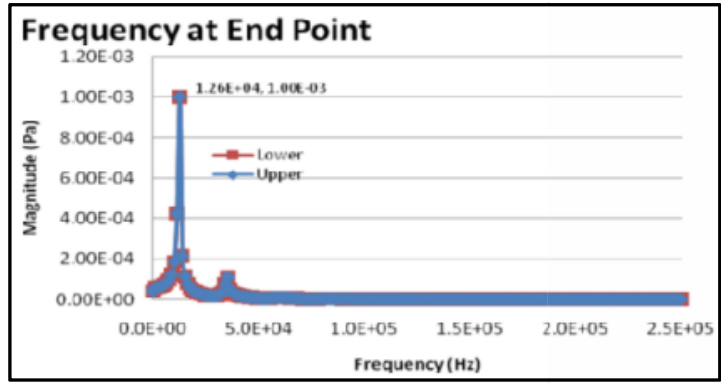

(d) End Point

Figure 7. Spectral density of 2D model (a) inlet - upper (b) inlet - lower (c) center point, and (d) end point 
Figure 8 show spectral density of 3D model at (a) inlet - upper (b) inlet - lower (c) center point (d) end point. Frequency at the inlet-upper, inlet-lower, center point, and end point of the membrane are occurred at the same frequency of $10.1 \mathrm{kHz}$. This indicates that the analysis numerical of the membrane using 3D model has resonance frequency around $10.1 \mathrm{kHz}$ during simulation of 8.00E-04 second.

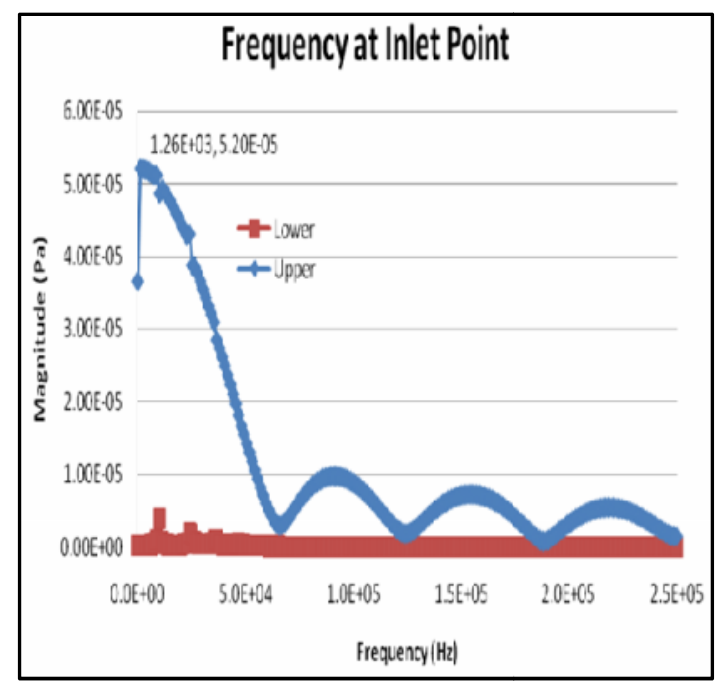

(a) Inlet - Upper

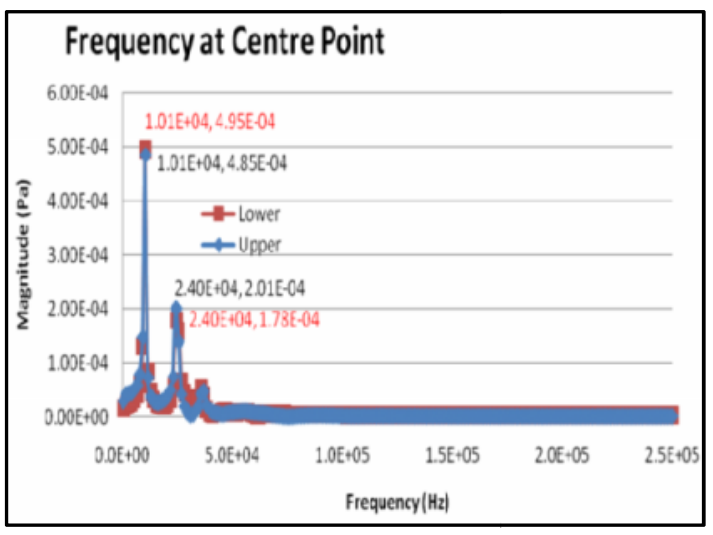

(c) Center Point

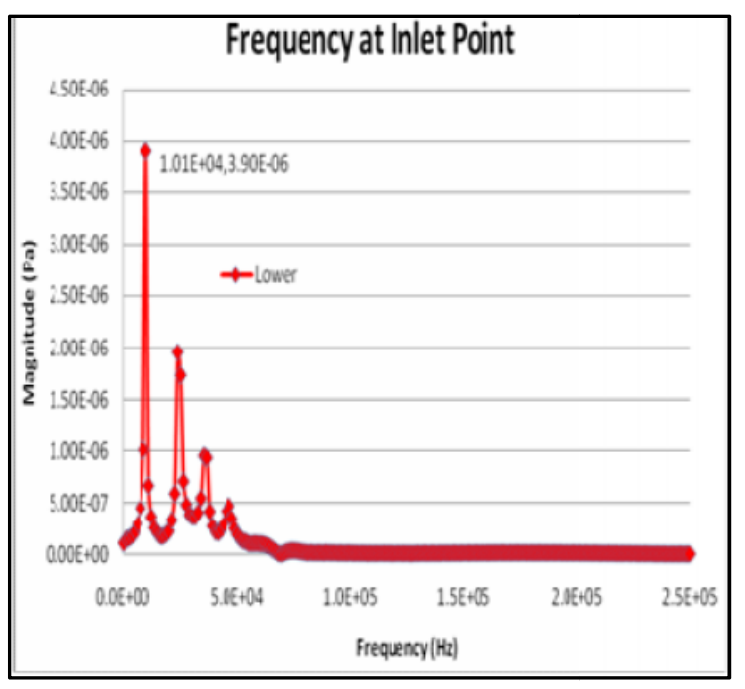

(b) Inlet - Lower

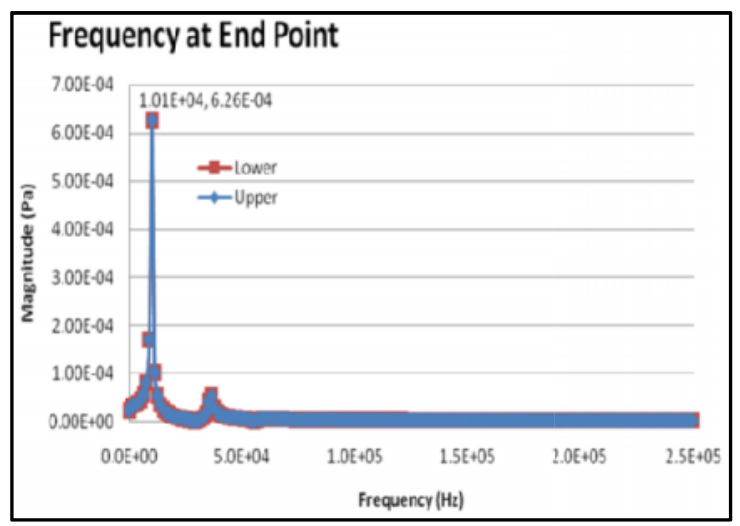

(d) End Point

Figure 8. Spectral density of 3D model (a) inlet - upper (b) inlet - lower (c) center point, and (d) end point

\section{CONCLUSIONS}

It is considered that this phenomenon is caused by the local resonance frequency of the membrane which varies along the longitudinal direction due to its trapezoidal shape. Based on the numerical method developed here, the basic characteristic of the membrane is obtained. The result shows that the frequency selectivity of the membrane is detected on the membrane surface. This indicates that the analysis numerical of the membrane using 2D and 3D model is effective to predict the frequency selectivity. The resonance frequencies are detected in the 2D and 3D model of $12.6 \mathrm{kHz}$ and $10.1 \mathrm{kHz}$, respectively. According to this result, the simulation program also can 
International Journal of Computer Science, Engineering and Applications (IJCSEA) Vol.2, No.4, August 2012

be applied to predict the pattern of pressure distribution and pressure changing on the membrane. This prediction can be used for the next step using experimental method.

\section{ACKNOWLEDGEMENTS}

The author would like to acknowledge Kawano Laboratory members at Osaka University Japan and the support of LPPI Tarumanagara University.

\section{REFERENCES}

[1] Békésy, von G., Current Status of Theories of Hearing, Science, 123 - 3201, 497 - 512, 1956.

[2] Békésy, von G., Experiments in Hearing, Mc Graw Hill, New York, 1960.

[3] Zwislocki, Josef J., Auditory sound trans-mission: an autobiographical perspective, Lawrence Erlbaum Associates Inc., New Jersey, USA, 2002.

[4] Moller, Aage R., Hearing, second edition : Anatomy, Physiology and disorders of the auditory system, 2 nd edition, Elsevier, USA, 2006

[5] Tu Jiyuan, Guan H. Yeoh, Chaogun Liu, Computational Fluid Dynamics : A Practical Approach, 1st Edition, Elsevier, USA, 2008.

[6] Versteeg H K and Malalasekera W, An Introduction to Computational Fluid Dynamics, second Edition, British:Prentice Hall, 2007.

[7] Batchelor G K, An Introduction to Fluid Dynamics, United States:Cambridge, 2007.

[8] Ferziger Joel H., Peric, Milovan, Computational Methods for Fluid Dynamics, third rev. edition. Germany:Springer, 2002.

[9] Ward A.J. - Smith, Internal Fluid Flow- The Fluid Dynamics of Flow in Pipes and Ducts, Great Britain:Pitman Press, 1980.

[10] Fangyi Chen, Howard Cohen, Thomas G. Bifano, Jason Castle, Jeffrey Fortin, Christopher Kapusta, David C. Mountain, Aleks Zosuls, and Allyn E. Hubbar, A hydromechanical biomimetic cochlea: Experiments and models, J. Acoust. Soc. Am. Volume 119, Issue 1, pp. 394-405, 2006.

[11] Liu Bo, Xiu L. Gao, Hong X. Yin, Shu Q. Luo, Jing Lu, A Detailed 3D Model of The Guinea Pig Cochlea, Brain Struct Funct (2007) 212:223-230, Springer-Verlag 2007.

[12] Tanaka, K., M. Abe, S. Ando, A novel mechanical cochlea "fishbone" with dual sensor/actuator charateristics, IEEE/ASME Transactions on Mechatronics 3 (2) 98-105, 1998.

[13] White R. D., K. Grosh, Microengineered hydromechanical cochlear model, Proceedings of the National Academy of Sciences of the United States of America 102 (5), 1296-1301, 2005.

[14] Anderson J. David, Computational Fluid Dynamics, Mc Graw-Hill Science, 1995.

[15] Steele, Charles R. and Taber, Larry A., Comparison of WKB Calculations and Experimental Results for Three-Dimensional Cochlear Models, J. Acoustical Society of America, 65(4), April 1979.

[16] Wittbrodt, M., S. Puria, and C.R. Steele, Developing a physical model of the human cochlea using micro-fabrication methods, Audiology and Neurotology 11(2):104-112, 2006.

\section{Authors}

Harto Tanujaya received S.T., M.T., and Ph.D. degrees in Mechanical Engineering from Tarumanagara University (in 1996), University of Indonesia (in 1999) and Osaka University (in 2010), respectively. He is a lecturer and researcher at Tarumanagara University and his fields of research are includes artificial cochlea, vibrations, and computational fluid dynamics (CFD). Recently, his current research project is vibration of the artificial basilar membrane using specific

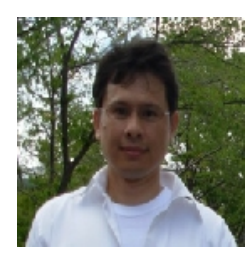
materials. 